\title{
A NOTE ON STOCHASTIC APPROXIMATION
}

J. R. BLUM ${ }^{1}$

1. A theorem on convergence of a sequence of random variables is proved in $\S 2$. In $\S 3$ this theorem is applied to prove convergence of a class of stochastic approximation procedures. The author was unable to verify whether the theorem of $\S 3$ could be derived from the general convergence theorem for stochastic approximation procedures due to Dvoretzky [1]. At any rate the method of proof used here appears to have some independent interest.

2. Let $\left\{X_{n}\right\}$ and $\left\{Y_{n}\right\}$ be infinite sequences of random variables. Let $I\{\cdots\}$ be the indicator (set characteristic function) of the set in brackets and for any random variable $U$ let $U^{+}$and $U^{-}$be the positive and negative part, respectively, of $U$. Then we have

Theorem 1. Suppose

$$
X_{n}-\sum_{j=1}^{n} Y_{j} \text { converges with probability one (w.p. 1). }
$$

(2.2) For some positive integer $k$ and every $\epsilon>0$

$$
\sum_{n=k}^{\infty} I\left\{X_{n} \geqq \epsilon, \cdots, X_{n-k+1} \geqq \epsilon\right\} Y_{n}^{+}<+\infty w . p .1
$$

and

$$
\sum_{n=k}^{\infty} I\left\{X_{n} \leqq-\epsilon, \cdots, X_{n-k+1} \leqq-\epsilon\right\} Y_{n}^{-}>-\infty \text { w.p. } 1 .
$$

Then $X_{n}$ converges w.p. 1 if and only if $\lim _{n \rightarrow \infty} Y_{n}=0$ w.p. 1 .

Proof. The necessity is immediate from (2.1). To prove sufficiency assume that we restrict ourselves to sample sequences in the set of probability one defined by the hypotheses of the theorem. Now if $\left\{X_{n}\right\}$ is any such sequence and $\lim _{n \rightarrow \infty} X_{n}=+\infty$ then, from (2.1), $\sum_{n=1}^{\infty} Y_{n}=+\infty$. But this clearly violates the first part of (2.2) for arbitrary $\epsilon>0$. Similarly if $\lim _{n \rightarrow \infty} X_{n}=-\infty$. Hence $P\left\{\lim _{n \rightarrow \infty} X_{n}\right.$ $=+\infty\}=P\left\{\lim _{n \rightarrow \infty} X_{n}=-\infty\right\}=0$. Now suppose $\left\{X_{n}\right\}$ is a sample sequence with $\lim \inf _{n \rightarrow \infty} X_{n}<\lim \sup _{n \rightarrow \infty} X_{n}$. Assume $\lim \sup _{n \rightarrow \infty} X_{n}$ $>0$ and choose numbers $a$ and $b$ with $\lim _{\inf _{n \rightarrow \infty}} X_{n}<a$ and $0<a<b$

Received by the editors July 31, 1957.

${ }^{1}$ Sponsored by The Office of Ordinance Research, U. S. Army, under Contract No. DA-33-008-ORD-965. 
$<\lim \sup _{n \rightarrow \infty} X_{n}$. Then it follows from the hypotheses that there are infinitely many pairs of positive integers $\left(n_{1}, n_{2}\right)$ satisfying

(i) $n_{1}<n_{2}$,

(ii) $X_{n} \geqq a / 2, \cdots, X_{n-k+1} \geqq a / 2$ for $n_{1} \leqq n \leqq n_{2}$,

(iii) $X_{n_{2}} \geqq b, X_{n_{1}} \leqq a$.

Then $X_{n_{2}}-X_{n_{1}} \geqq b-a$ and it follows from (2.1) that $\sum_{j=n_{1}+1}^{n_{2}} Y_{j}$ $\geqq(b-a) / 2$ for $n_{1}$ sufficiently large. On the other hand if we apply the first part of (2.2) with $\epsilon=a / 2$ we find that $\sum_{j=n_{1}+1}^{n_{2}} Y_{j}^{+}$approaches zero as $n_{1}$ increases. Consequently we have a contradiction. A similar argument applies to those samples sequences for which lim $\sup _{n \rightarrow \infty} X_{n}$ $\leqq 0$, and the theorem is proved.

3. For each real number $x$ let $U_{x}$ be an integrable random variable with distribution function $H(u \mid x)$ and mean value $M(x)$. In many problems it is of interest to find a root of the equation $M(x)=0$ assuming that the distribution functions $H(u \mid x)$ and the function $M(x)$ are unknown but that one may make observations on the random variable $U_{x}$ for any desired value $x$. To this end Robbins and Monro [2] proposed a stochastic approximation scheme given by

$$
x_{n+1}=x_{n}+a_{n} u_{n}
$$

where $x_{1}$ is an arbitrary number, $\left\{a_{n}\right\}$ is a sequence of positive numbers and $u_{n}$ is a random variable distributed according to $H\left(u \mid x_{n}\right)$. Robbins and Monro showed that under appropriate conditions $x_{n}$ converges in probability to a root of the equation $M(x)=0$. Much further work has since been done on this problem. For a description of this work and a list of references see Derman [3]. In this paper we shall consider a class of stochastic approximation procedures yielding estimates which converge with probability one to a root of the equation $M(x)=0$.

Consider now the following set of conditions on the stochastic structure involved.

$$
\begin{aligned}
& |M(x)| \leqq M<\infty . \\
& \int_{-\infty}^{+\infty}[u-M(x)]^{2} d H(u \mid x) \leqq \sigma^{2}<\infty . \\
& M(x)<0 \text { for } x<\beta, \quad M(x)>0 \text { for } x>\beta . \\
& \operatorname{Inf}_{x \in I}|M(x)|>0 \text { for every closed bounded interval } I \text { not con- } \\
& \text { taining } \beta .
\end{aligned}
$$

The generalization of the Robbins-Monro scheme we have in mind is motivated by the idea that one may not wish to take the $n$th 
observation from the distribution $H\left(u \mid x_{n}\right)$ but instead from the distribution $H\left(u \mid z_{n}\right)$ where $z_{n}$ is a point determined in same fashion from a number of previous observations. To this end we consider for each positive integer $n$ two measurable functions $f_{n}\left(x_{1}, \cdots, x_{n}\right)$ and $g_{n}\left(x_{1}, \cdots, x_{n}\right)$. Assume that the functions $f_{n}\left(x_{1}, \cdots, x_{n}\right)$ and $g_{n}\left(x_{1}, \cdots, x_{n}\right)$ satisfy: (3.5) There exists a positive integer $k$ and for every real number $r$ and every positive number $\eta$ a positive integer $n(r, \eta)$ such that $n \geqq n(r, \eta), x_{n} \geqq r+\eta, \cdots, x_{n-k+1} \geqq r+\eta$ implies $f_{n}\left(x_{1}, \cdots, x_{n}\right)>r$ and $n \geqq n(r, \eta), x_{n} \leqq r-\eta, \cdots, x_{n-k+1} \leqq r-\eta$ implies $f_{n}\left(x_{1}, \cdots, x_{n}\right)<r$.

(3.6) $\sum_{n=1}^{\infty}\left|g_{n}\left(x_{1}, \cdots, x_{n}\right)\right|<\infty$ for every sequence $\left\{x_{n}\right\}$ of real numbers.

Let $\left\{a_{n}\right\}$ be a sequence of positive numbers satisfying

$$
\sum_{n} a_{n}=\infty, \quad \sum_{n} a_{n}^{2}<\infty .
$$

Let $x_{1}$ be an arbitrary number. Define $x_{n+1}$ recursively by the equation

$$
x_{n+1}-x_{n}=g_{n}\left(x_{1}, \cdots, x_{n}\right)-a_{n} u_{n}
$$

where $u_{n}$ is an observation on the random variable distributed according to $H\left(u \mid f_{n}\left(x_{1}, \cdots, x_{n}\right)\right)$. Then we have

THEOREM 2. If $\left\{x_{n}\right\}$ is the process (3.8) and if conditions (3.1) through (3.7) hold then $P\left\{\lim _{n \rightarrow \infty} x_{n}=\beta\right\}=1$.

Proof. We shall assume without loss of generality that $\beta=0$. We prove the theorem by first showing that Theorem 1 applies and then verifying that the resulting limit is the correct one. To do this we make the following identifications with the random variables of Theorem 1: Let $X_{0}=0, Y_{1}=0, X_{n}=x_{n}$, and $Y_{n}=g_{n-1}\left(x_{1}, \cdots, x_{n-1}\right)$ $-a_{n-1} M\left(f_{n-1}\right)$. Then

$$
X_{n}-\sum_{j=1}^{n} Y_{j}=\sum_{j=1}^{n}\left[\left(X_{j}-X_{j-1}\right)-Y_{j}\right]=\sum_{j=1}^{n-1} a_{j}\left[M\left(f_{j}\right)-u_{j}\right] .
$$

Now from (3.2) and (3.7) we have that $\sum_{n} a_{n}^{2} E\left\{\left[M\left(f_{j}\right)-u_{j}\right]^{2}\right\}$ $\leqq \sigma^{2} \sum_{n} a_{n}^{2}<\infty$ and it follows from straight forward considerations (see e.g. Loeve $\left[2\right.$, p. 387]) that (2.1) holds. Now if $x_{n} \geqq \epsilon, \cdots$, $x_{n-k+1} \geqq \epsilon$ then we know from (3.5) that for $n$ sufficiently large $f_{n}\left(x_{1}, \cdots, x_{n}\right)>0$. This together with (3.3) and (3.6) establishes the first part of (2.2) and a similar argument takes care of the second part. Now 


$$
\begin{aligned}
\left|Y_{n}\right| & =\left|g_{n-1}\left(x_{1}, \cdots, x_{n-1}\right)-a_{n-1} M\left(f_{n-1}\right)\right| \\
& \leqq\left|g_{n-1}\left(x_{1}, \cdots, x_{n-1}\right)\right|+a_{n-1} M
\end{aligned}
$$

and from (3.1), (3.6), and (3.7) we see that $\lim _{n \rightarrow \infty} Y_{n}=0$ w.p. 1 . Hence Theorem 1 applies. Now suppose $P\left\{\lim _{n \rightarrow \infty} x_{n}=0\right\}<1$. Then there exist numbers $a$ and $b$ with (say) $0<a<b$ such that $P\left\{a \leqq \lim _{n \rightarrow \infty} x_{n} \leqq b\right\}>0$. Then from (3.5) it follows that for any such sample sequence $\left\{x_{n}\right\}$ we have $0<a \leqq \lim _{n \rightarrow \infty} f_{n}\left(x_{1}, \cdots, x_{n}\right)$ $=\lim _{n \rightarrow \infty} x_{n} \leqq b$. It follows from (3.3), (3.4), and (3.6) that

$$
\sum_{n=1}^{\infty}\left[g_{n}\left(x_{1}, \cdots, x_{n}\right)-a_{n} M\left(f_{n}\right)\right]=-\infty .
$$

But then $\sum_{n=1}^{\infty} a_{n}\left[M\left(f_{n}\right)-u_{n}\right]=+\infty$ and this happens with probability zero. Consequently $P\left\{\lim _{n \rightarrow \infty} x_{n}=0\right\}=1$.

We mention in passing that this same type of modification may be applied to the stochastic approximation procedure discussed by Kiefer and Wolfowitz [5] which estimates the point where the regression function achieves a maximum.

\section{REFERENCES}

1. A. Dvoretzky, On stochastic approximation, Proceedings of the Third Berkeley Symposium of Mathematical Statistics and Probability, vol. 1, 1956, pp. 39-55.

2. H. Robbins and S. Monro, A stochastic approximation method, Ann. Math. Stat. vol. $22(1951)$ pp. 400-407.

3. C. Derman, Stochastic approximation, Ann. Math. Stat. vol. 27 (1956) pp. 879886.

4. M. Loeve, Probability theory, Van Nostrand, 1955.

5. J. Kiefer and J. Wolfowitz, Stochastic estimation of the maximum of a regression function, Ann. Math. Stat. vol. 23 (1952) pp. 462-466.

INDIANA UNIVERSITY 